pneumonia, and in cases of intolerance, dapsone or pentamidine can be used as alternative treatment options.

Methods Retrospective review of patient notes and electronic patient records, for those patients who have completed treatment for Acute Lymphoblastic Leukaemia on the UKALL2003 protocol at the Royal Marsden Hospital.

Results 164 patients were commenced on co-trimoxazole, and one was commenced on dapsone. $22(13.4 \%)$ of patients did not tolerate co-trimoxazole, with 20 cases of intolerance secondary to cytopenia.

Of the 22 patients who discontinued co-trimoxazole, 20 patients $(87 \%)$ have documentation of G6PD status being investigated, all of whom were negative for G6PD deficiency.

The first 5 patients to require 2 nd line therapy were commenced on pentamidine, with 'cytopenia' cited as the cause of co-trimoxazole intolerance for all of these patients. One patient was subsequently changed onto dapsone.

17 patients were commenced on dapsone as 2 nd line therapy for PCP prophylaxis, with 'cytopenia' cited as the cause of co-trimoxazole intolerance in 15 patients.

5 of the 17 patients who were commenced on dapsone as 2 nd line treatment, did not tolerate dapsone. Of these five patients, one had persistent neutropenia, two developed methaemaglobinaemia, and two developed dapsone syndrome.

Only one of 165 patients was suspected of developing pneumocystis pneumonia, and retrospectively this patient was found to be non-compliant with co-trimoxazole.

Conclusions Current PCP prophylaxis treatments are effective, and for the majority of patients, co-trimoxazole is well tolerated.

\section{LATE EFFECTS IN SURVIVORS OF INFANT LEUKEMIA IN SINGLE CENTER}

doi:10.1136/archdischild-2012-302724.0767

'S Ansari, 'A Shir Ali, ${ }^{2}$ S Ziaie, 'P Vossough. 'Tehran University of Medical Sciences; ${ }^{2}$ Shahid Beheshti University of Medical Sciences, Tehran, Iran

Background Acute lymphoblastic leukemia (ALL) is the most common childhood malignancy, accounting for $30 \%$ of all cancers occurring in childhood .long term sequel of treatment are now being reported. Children who survive acute lymphoblastic leukemia are at risk for leukemia-related or treatment-related complications.

Methods In this study we evaluated 66 patients with ALL have survived for more than5 years after diagnosis. Long-term squeal of treatment, such as impaired intellectual and psychomotor functioning, neuroendocrine abnormalities, impaired reproductive capacity, cardio toxicity, and second malignant neoplasm's, are being reported.

Results of the 66 patients, 43 cases were male and 23 female. Mean age was $14.59 \pm 4.36$ (range 10-25 years). 42 patients received chemotherapy alone, 24 patients who received chemotherapy and CNS radiation therapy. Short height $33 / 3 \%$,over weight $50 \%$, low bone density $53 \%$, learning disabilities $6 / 1 \%$, hyperthyroidism $1 / 5 \%$, sexual development (pubertal delay) $7 / 6 \%$, over weight are more common in children who get chemotherapy without radiotherapy. $31 / 8 \%$ of patients don't have late effects. $30 / 3$ had at least one late complication.

Conclusion These results indicate that late sequelae are common in long term survivors of infant leukemia and are often related to CRT the most common problem are short stature and over weight.

\section{EVALUATION OF SERUM TRANSFERRIN RECEPTOR IN PATIENTS AFFECTED BY IRON-DEFICIENCY ANEMIA AND COMPARISON WITH CONTROL GROUP}

doi:10.1136/archdischild-2012-302724.0768
'S Shams, ${ }^{2} \mathrm{SO}$ Mohammadian, 'M Monajemzadeh, ${ }^{2} \mathrm{H}$ Irani, ${ }^{2} \mathrm{~L}$ Shafaghat, 'MT Haghi Ashtiani. 'Pathology Department; ' $C$ enter of Excellence for Pediatrics, Children's Medical Center, Tehran University of Medical Sciences, Tehran, Iran

Background and Aims Iron deficiency anemia is one of the most common nutritional deficiencies and public health around the world. The growing Children are one of groups that are at high risk for this problem. Iron deficiency anemia can cause mental, motor or behavior problems. So early diagnosis and treatment can prevent great side effects. One of the diagnosis means is measuring of soluble tranferrin receptor level (sTfR) in serum because level of this receptor increase in result of iron depletion in iron deficiency but has no change in other.

Methods and materials: This was a case control study included 64 children with iron deficiency anemia (IDA) and healthy subjects. The study conducted, in 2008-2010 in Children Medical Center. Blood samples collected from every patient in case and control groups and $\mathrm{Hb}, \mathrm{MCV}, \mathrm{Fe}$, Ferritin, TIBC and sTfR measured and compared in the groups.

Results Compared to the control group, serum sTfR mean level was significantly higher in children with IDA than control group $(1.87 \mathrm{vs} .1 .06 \mu \mathrm{g} / \mathrm{ml}, \mathrm{P}$ value $=0.002) . \mathrm{sTfR}$ showed negative correlations with $\mathrm{Hb}(\mathrm{r}=-0.629, \mathrm{p}<0.001), \mathrm{MCV}(\mathrm{r}=-0.649, \mathrm{p}<0.001)$ and serum Ferritin $(r=-0.224, p=0.053)$, although it was not significant for Ferritin. There was no significant differences between cases and controls in sTfR mean level with regard to gender and age categories. $(\mathrm{P}>0.05)$.

Conclusions This study shows sTfR level can be an appropriate biomarker for diagnosis of IDA, particularly in patient with IDA coexisting with inflammation.

\section{EXCESSIVE MENSTRUAL BLEEDING IS A RISK FACTOR OF ANEMIA IN ADOLESCENT SCHOOLGIRLS}

doi:10.1136/archdischild-2012-302724.0769

I Tarasova, V Chernov. Child Hematology and Oncology, Federal Research Center of Pediatric Hematology, Oncology, and Immunology named after Dmitry Rogachev, Moscow, Russia

Background and Aims Adolescents form a group at risk of iron deficiency development. An additional factor leading to depletion of iron stores in adolescent girls is blood loss during menstrual bleeding.

Methods A cross-sectional nonrandomized descriptive study was carried out in order to evaluate the incidence of dysfunctional uterine bleeding (DUB) in adolescent girls and detect the relationship between menstrual blood loss and anemia, in order to develop measures to improve the health status of adolescents. The study was carried out within the routine health examination of schoolchildren in Vologda. A total of 1340 girls aged 10-18 years (mean age $13.81 \pm 0.04$ years) were selected at random. The menstrual function was studied in 883 girls aged $11-18$ years (mean age $14.5 \pm 0.04$ years) by the data of questionnaires and results of gynecological examinations.

Results The prevalence of anemia ( $\mathrm{Hb}$ less than $120 \mathrm{~g} / \mathrm{l}$ ) in all examined girls of Vologda was $10.7 \%(n=143)$, in adolescent girls with menses $-10.5 \%(n=93)$. The age of menarche varied from 10 to 16 years (mean age $12.57 \pm 0.03$ years). DUB were detected in 54 (6.2\%) girls. Anemia was detected in 11 (20.4\%) girls with DUB. Of the menstrual characteristics excessively heavy menstrual bleeding was the only risk factor for anemia $(\mathrm{OR}=0.43,95 \% \mathrm{CI}=0.22-0.87$, $p=0.0348$ ).

Conclusions Menstrual iron loss is the main risk factor for iron deficiency development, and hence, in order to detect the causes of anemia in females of reproductive age the complete anamnesis on the pattern of menstruation should be collected. 


\section{RISK FACTORS ASSOCIATED WITH ANEMIA AND NUTRITIONAL STATUS IN INFANTS}

doi:10.1136/archdischild-2012-302724.0770

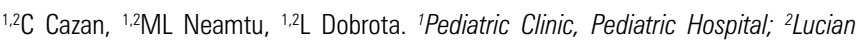
Blaga University of Sibiu, Sibiu, Romania

Background Growth assessment is an integral part of infants health. An understanding of anemia risk factors is essential to identify the groups that are more vulnerable.

The Aim of the study was to identify the risk factors for malnutrition in the vulnerable age group of 6 to 12 months and to evaluate possible related risk factors with anemia.

Methods The cross-sectional study which evaluated 206 infants aged between 6-12 months who attended the Pediatric Clinic during the years 2010-2011 for inadequate growth. Mothers were interviewed to collect informations regarding socioeconomic status and nutrition practices. Nutritional status was evaluated by anthropometric measurements using growth charts. Anemia was diagnosed if hemoglobin was $\leq 11 \mathrm{~g} / \mathrm{dL}$.

Results Mild and moderate anemia was characterised by hemoglobin levels below 11.0 and $9.5 \mathrm{~g} / \mathrm{dL}$. Rates for mild and moderate anemia were $38.6 \%$ and $11.9 \%$. The highest anemia prevalence was found at 6 to 8 months of age. The risk factors for anemia were: urban residence $(p=0.004)$, fever in the past 5-7 days $(p<0.001)$ and age at $6-8$ months $(p=0.024)$, socioeconomic level and nutrition practices. Infants who were exclusively breastfed for 6 months showed lower prevalence of anemia compared to their mixed feeding. According to weight and length for-age, $49 \%$ of the infants were at $25^{\text {th }}, 32 \%$ at $10^{\text {th }}, 9.2 \%$ at $5^{\text {th }}$ and there was a significant correlation between the duration of breastfeeding and nutritional status.

Conclusions Strategies to control infant anemia should include health promotion and nutritional education for families from all socioeconomic levels.

\section{HIGH INCIDENCE OF IRON DEFICIENCY IN YOUNG CHILDREN WITH CYSTIC FIBROSIS}

doi:10.1136/archdischild-2012-302724.0771

L Uijterschout, J Vloemans, M Nuysink, DM Hendriks, F Brus. Juliana Children's Hospital/HAGAZiekenhuis, The Hague, The Netherlands

Background Iron deficiency (ID) is common in patients with cystic fibrosis (CF). In adult CF patients ID is related to lung disease severity and thought to be caused by chronic inflammation. Increased iron levels in sputum are associated with P. aeruginosa infections.

Aim To establish the prevalence of ID and iron deficiency anemia (IDA) in children with CF and associations of ID with dietary iron intake, lung disease severity and Pseudomonas aeruginosa infection.

Methods Clinical charts of 54 children with CF aged 0 to 16 were reviewed. Follow-up varied from 1 to 14 years with 346 annual observations in total. Laboratory data (hemoglobin $(\mathrm{Hb})$, serum ferritin (SF)) and results of pulmonary function tests, sputum cultures and 3-day food records were collected.

Results 46 children $(85.2 \%)$ were iron deficient $(\mathrm{SF}<30 \mu \mathrm{g} / \mathrm{l})$ in at least one year and ID was present in 329 of 346 observations (95.1\%). IDA ( $\mathrm{SF}<30 \mu \mathrm{g} / \mathrm{l}$ and $\mathrm{Hb}>2 \mathrm{SD}$ below the mean of similarly aged children) was present in 8 observations $(2.4 \%)$ in 6 patients $(11.1 \%)$. Children with ID were younger (6.4 year versus 10.6 year, $p=0.00$ ) and had less pulmonary exacerbations ( $p=0.01$ ). ID was not associated with FEV1, Pseudomonas aeruginosa infection or dietary iron intake.

Conclusion ID is common in young children with CF and associated with less pulmonary exacerbations. We suggest that ID in these children is caused by rapid growth and accelerated erythropoies instead of disease severity or insufficient dietary iron intake.

\section{SERUM HEPCIDIN IN CHILDREN WITH BETA-THALASSEMIA}

doi:10.1136/archdischild-2012-302724.0772

${ }^{1} \mathrm{HM}$ Assem, ${ }^{2} \mathrm{OA}$ Sharaki, ${ }^{3} \mathrm{ME}$ El-Shennawi, ${ }^{3} \mathrm{HM}$ Gomaa. 'Pediatrics, Faculty of Medicine, Beirut Arab University, Beirut, Lebanon; ${ }^{2}$ Clinical Pathology; ${ }^{3}$ Pediatrics, Faculty of Medicine - Alexandria University, Alexandria, Egypt

Background Hepcidin, first described about 10 years ago, is a key iron - regulatory hormone. However, hepcidin measurement in a variety of human disease states are still lacking.

Aim To study serum level of hepcidin hormone in children with beta-thalassemia major (TM) and intermedia (TI)

Subjects and Methods The work was conducted on 50 children divided into 3 groups: 15 children with beta-thalassemia major, 10 children with beta-thalassemia intermedia, and 25 healthy children as a control group.

Thalassemic children included in the study were subjected to: Detailed history taking, clinical examination and measurement of serum hepcidin hormone level by (ELISA).

Results The mean serum hepcidin level was significantly higher in children with TM than in patients with TI and the controls. The ratio of serum hepcidin to serum ferritin in TI was significantly lower than those with TM. In addition, there was a significant positive relation between serum hepcidin and serum ferritin and also with serum iron.

Conclusions Hepcidin measurement may be useful as part of the diagnostic and prognostic evaluation of thalassemia as it may allow a more accurate assessment of the degree of iron overload and the maldistribution of iron.

In the future, it may be possible to use exogenous hepcidin to restore normal iron homeostasis in patients with thalassemia especially thalassemia intermedia.

\section{PLATELETS ANTIBODIES AND SERUM LEPTIN IN CHILDHOOD IMIMUNOTHROMBOCYTOPENIC PURPURA}

doi:10.1136/archdischild-2012-302724.0773

${ }^{1}$ HB Hamed, ${ }^{2}$ AM Zahran. 'Clinical Pathology, South Egypt Cancer Institute Assiut University; ${ }^{2}$ Clinical Pathology, Assiut University, Assiut, Egypt

Background and Aim Platelets antigens, Anti-platelets antibodies, serum leptin measurement may be important in defining the pathogenesis of thrombocytopenic states.

Methods In this study we measured the platelets CD41, CD61, CD62P, Platelets IgG, IgM by flowcytometry and serum leptin by ELISA of 20 children diagnosed as ITP and 20 normal children as control.

Results We observed That there were no significant difference in white blood cells count, hemoglobin concentration between ITP patients and controls. Platelets count was significantly decreased, and mean platelet volume (MPV) was significantly increased in patients than controls $\mathrm{P}=0.000$. The percentage of $\mathrm{CD} 41$ expressing platelets was significantly lower in ITP children compared to controls $(\mathrm{P}=0.001)$ but the percentage of $\mathrm{CD} 61$ expressing platelets was not significantly different between ITP patients and controls. Platelet activation marker CD62P was significantly expressed in patients than controls (0.000). Furthermore, the amount of CD62P per cell, represented by the MFI was significantly higher in patients than controls (0.000). The percentage of platelets associated IgM and IgC were was significantly increased in patients than controls $(\mathrm{P}=0.000)$ Also the MFI of IgM and IgG were significantly higher in patients than controls .Finally the concentration of serum leptin was increased in patients than controls $(\mathrm{P}=0.000)$ (table 2$)$. There was a negative correlation between The platelets count and Platelets IgG $(\mathrm{P}=0.000$ and $\mathrm{r}=-0.88)$.

Conclusion We concluded that The demonstration of antiplatelet antibodies (PAIgG, PAIgM) and decreased detection of platelet 